\title{
Inadvertent Application of a Drug Mixture into the Subarachnoid Space When Performing an Outward-Going Lumbar Intervertebral Foramina Injection
}

\author{
Fachuan Nie*, Suxia Wang, Jian Cui \\ Pain Department of Southwest Hospital, Third Military Medical University, Chongqing, China \\ Email: *Fachuan_nie@sina.com, lizhongsusu@163.com, cqjian211@163.com
}

Received 23 February 2016; accepted 1 May 2016; published 4 May 2016

Copyright (C) 2016 by authors and Scientific Research Publishing Inc.

This work is licensed under the Creative Commons Attribution International License (CC BY). http://creativecommons.org/licenses/by/4.0/

(c) (i) Open Access

\begin{abstract}
An outward-going intervertebral foramina puncture along the side of lumbar vertebra spinous process was used to avoid infusing drugs into the subarachnoid space. However, we still had an inadvertent malpractice. The patient was diagnosed with lumbar discogenic lower back pain and was administrated an outward-going intervertebral foramina puncture through the side of lumbar vertebra spinous process $\left(L_{5}-S_{1}\right)$. The drug mixture, containing triamcinolone acetonide suspension, cyanocobalamin and bupivacaine $\mathrm{HCl}$, was injected. The patient had palpitations, chest tightness, dyspnea, bradycardia, and hypotension $3 \mathrm{~min}$ right after the injection. The vital signs gradually stabilized after treatment with atropine sulfate; however, he had complete sensorimotor loss below the level of distribution of the $T_{2}$ spinal nerve. The cerebrospinal fluid pressure was lower in the subarachnoid space and the cerebrospinal fluid was slightly turbid and light pink in color. Six hours later, the sensory, motor, defecation, and urination functions returned to normal level. The patient had follow-up evaluations at 1, 3, and 6 months. The lumbar and hip pain was mild-to-severe at times as usual, but there were no other neurologic sequelae. Conclusions: This case showed that there is a risk that drugs may stray into the subarachnoid space during an outward-going intervertebral foramina puncture through the side of lumbar vertebral spinous process. The complication may occur due to a distal nerve root cyst. The result of triamcinolone acetonide suspension and cyanocobalamin injection into the cerebrospinal fluid was not as serious as we imagined.
\end{abstract}

\section{Keywords}

Interventional Therapy, Paravertebral Injection, Pain Treatments, Accident, Subarachnoid Space,

\footnotetext{
${ }^{*}$ Corresponding author.
}

How to cite this paper: Nie, F.C., Wang, S.X. and Cui, J. (2016) Inadvertent Application of a Drug Mixture into the Subarachnoid Space When Performing an Outward-Going Lumbar Intervertebral Foramina Injection. World Journal of Neuroscience, 6, 85-89. http://dx.doi.org/10.4236/wjns.2016.62011 


\section{Cerebrospinal Fluid}

\section{Introduction}

It is a common technique to inject local anesthetics and anti-inflammatory drugs for treatment of radicular neuritis caused by spinal degeneration in the clinic field of chronic pain. Main safety control of the technique is to avoid the drugs being injected into the subarachnoid space. The injurious consequences induced by inadvertent infusion of medications into the cerebrospinal fluid vary based on the different drug compositions [1] [2]. Because of the number of outpatients, time constraints, and relatively simple safety control procedures, the risks that drugs are inadvertently introduced into the subarachnoid space during a lumbar intervertebral foramina injection are considerable. An outward-going intervertebral foramina puncture along lumbar vertebra spinous process was performed to avoid the risk. Over the past 5 years, more than 5000 cases have been performed by our physicians to treat pain and this technique was considered to be safe and effective. Nevertheless, we inadvertently introduced a drug mixture into the cerebrospinal fluid which contained glucocorticosteroid suspension, bupivacaine, and vitamin $\mathrm{B}_{12}$.

\section{Case Report}

The patient was 33-year-old male farmer. He complained of persistent waist and hip pain for 3 months. Pain in the right side was greater than that in the left side. Moreover, pain was aggravated by a long walk and slightly relieved after a brief rest. Additionally, the intermittent administration of anti-inflammatory analgesic was not effective. The patient did not complain of leg pain, numbness, or dysfunction. The physical examination revealed pain existed in the lower back and the upper inside quadrants of both hips. The Lasegue test and the Patrick sign were negative. The knee jerk reflex and Achilles tendon reflexes were normal. The Babinski sign was negative. Laboratory examinations showed normal Erythrocyte Sedimentation Rate, Blood Leukocyte and Neutrophil percentage. The compact tomography image showed narrowing disc space of $\mathrm{L}_{4} / \mathrm{L}_{5}$ and $\mathrm{L}_{5} / \mathrm{S}_{1}$ and modest middle right herniation of the $\mathrm{L}_{5} / \mathrm{S}_{1}$ disc. Based on the above evidence, the clinic diagnosis of "lumbar discogenic lower back pain” was obtained [3] [4].

After patient consent was obtained, we performed an outward-going intervertebral foramina puncture along the right side of lumbar vertebra spinous process $\left(\mathrm{L}_{5}-\mathrm{S}_{1}\right)$ to inject glucocorticoids and local anesthetic to treat the lower back pain under X-ray image guidance. The operator is a senior physician. The patient was placed in the left chest-knee horizontal position. The spinous process of $\mathrm{L}_{5}$ was determined according to anatomic landmarks. The puncture point was $1.5 \mathrm{~cm}$ right to the bottom edge of the $\mathrm{L}_{5}$ spinous process. The needle was inserted vertically to make contact with the lamina of lumbar vertebra, and the drug mixture (2 ml) was injected. The drug mixture contained triamcinolone acetonide suspension (20 mg), cyanocobalamin for injection (1 mg), bupivacaine $\mathrm{HCl}$ (37.5 mg), and physiologic saline [5] [6]. The total injection volume was 10 ml. The triamcinolone acetonide suspension for injection was obtained from Zhejiang Xianju Pharmaceutical Company (Xianju, Zhejiang Province, China) and the catalognumber was H33020762. Cyanocobalamin for injection was a product of Yaoyou Pharmaceutical Co, Ltd (Chongqing, China); the catalognumber was H20066849. Bupivacaine HCl was purchased from Southwest Pharmaceutical Limited (Chongqing, China); the catalog number was H50020018. Then we marked the depth of the needle, withdrew the needle back to the subcutaneous tissue, adjusted the needle outward-going to about 25 degree angle, inserted the needle to the marked depth, and continued $1 \mathrm{~cm}$ deeper. The X-ray examination identified the needle tip across the lamina and transverse process and arrived at the outer edge of the intervertebral foramen. The plunger was drawn back and no blood or cerebrospinal fluid returned in the syringe, then the drug mixture $(7 \mathrm{ml})$ was injected. Figure 1 shows a schematic diagram of the puncture procedure.

The patient had palpitations, chest tightness, dyspnea, limb weakness, and abdominal pain 3 min after the injection. The vital signs indicated that the heart rate was 50 beats/min and the upper arm blood pressure was $86 / 48 \mathrm{mmHg}$. The vein was accessed immediately and a single injection of atropine sulfate $0.5 \mathrm{mg}$ (catalognumber H43021679; Hunan Tianxin Pharmaceutical Co, Ltd, Liuyang, China) was administrated and intravenous drop infusion of another $1 \mathrm{mg}$ of atropine sulfate in $250 \mathrm{ml}$ of normal saline was administered slowly. The heart rate was gradually increased from 42 beats/min to 92 beats/min. The upper arm blood pressure gradually 


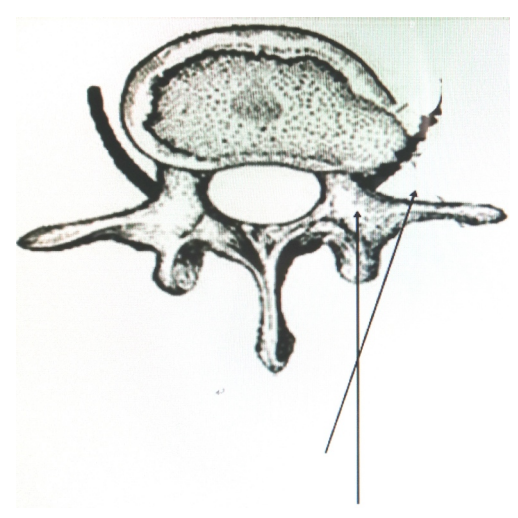

Figure 1. Schematic diagram of the outward-going puncture for the lumbar intervertebral foramina of $\mathrm{L}_{5} / \mathrm{S}_{1}$.

increased from 76/40 mmHg to 118/72 mmHg. The patient noted that the palpitations, chest tightness, and respiratory difficulties were significantly improved. The physical examination showed that the patient was in a conscious state; the heart and respiratory rates were stable; tactile sensation below the cutaneous innervation of $\mathrm{T}_{2}$ spinal nerve was completely absent; the muscle strength of the lower limbs was 0 of 5 (Lovett grade); the tactile sensation of the upper limbs was decreased; and the muscle strength of the upper limbs was 4 of 5 . The patient was admitted to the hospital $30 \mathrm{~min}$ later and was treated with dexamethasone sodium phosphate $20 \mathrm{mg}$ injection (catalognumber H50021043; Dikang Changjiang Pharmaceutical Company, Chongqing, China) via a slow intravenous drip.

Two hours later, the vital signs were stable. The loss of tactile sensation had descended approximately two thoracic nerve segment levels. The muscle strength of the upper limbs returned to normal; however, the muscle strength of the lower limbs remained at 0 . The patient was placed in the left chest-knee horizontal position to perform a subarachnoid puncture to examine the cerebrospinal fluid. An 18 G Tuohy needle (AS-E/S; Tuoren Company, Henan, China) was inserted into the spinous gap between $\mathrm{L}_{3}$ and $\mathrm{L}_{4}$. The loss of resistance served to confirm that the Tuohy needle was in the epidural cavity. Next, a $22 \mathrm{G}$ lumbar puncture needle was inserted into the subarachnoid cavity via the Tuohy needle. The cerebrospinal fluid pressure was $0-2 \mathrm{~cm} \mathrm{H}_{2} \mathrm{O}$. Three milliliters of cerebrospinal fluid was slowly aspirated. The cerebrospinal fluid was noted to be slightly turbid and light pink in color, which were consistent with the physical properties of the diluted mixture of triamcinolone acetonide acetate suspension and cyanocobalamin. After opening the puncture needle of the subarachnoid space for 1 min, only 1 drop of cerebrospinal fluid came out. Then, $5 \mathrm{ml}$ of normal saline was injected into the subarachnoid space. Finally, the double puncture needles were withdrew from the spinal column.

After $4 \mathrm{~h}$, the squeezing feeling in the chest and abdomen disappeared. The muscle strength and the tactile sensations of the lower limbs and perineal region gradually recovered.

Six hours later, the tactile sensations of the entire body were restored. The muscle strength of all limbs returned to normal. Defecation and urination were normal.

A magnetic resonance image (Magnetom; Prisma Siemens, Nuremberg, Germany) was performed to check the lumbar spine $22 \mathrm{~h}$ later, and revealed a lumbar disc herniation between $\mathrm{L}_{5}$ and $\mathrm{S}_{1}$. The nerve root adjacent to the intervertebral foramen was clearly visible, and there was no formation of a nerve root sheath cyst (Figure 2(L)). The transverse sections of the $\mathrm{L}_{4} / \mathrm{L}_{5}$ intervertebral discs showed that the water content of the lateral nerve root of the right intervertebral foramen was slightly increased (Figure 2(R)). It was the opinion of the radiologist that this finding was not sufficient to diagnose a nerve root cyst.

The patient had no unusual feelings and was discharged to home after a 3-day hospital stay. Telephone call follow-ups were made 1, 3, and 6 months after he returned home. The lumbar and hip pain was mild and severe at times as usual, but there were no other neurological abnormalities.

\section{Discussion}

Local anesthetics are administrated to produce nerve blocks and anti-inflammatory analgesics are injected into different locations to treat pain in outpatients. Both methods have high safety and efficacy profiles worldwide 


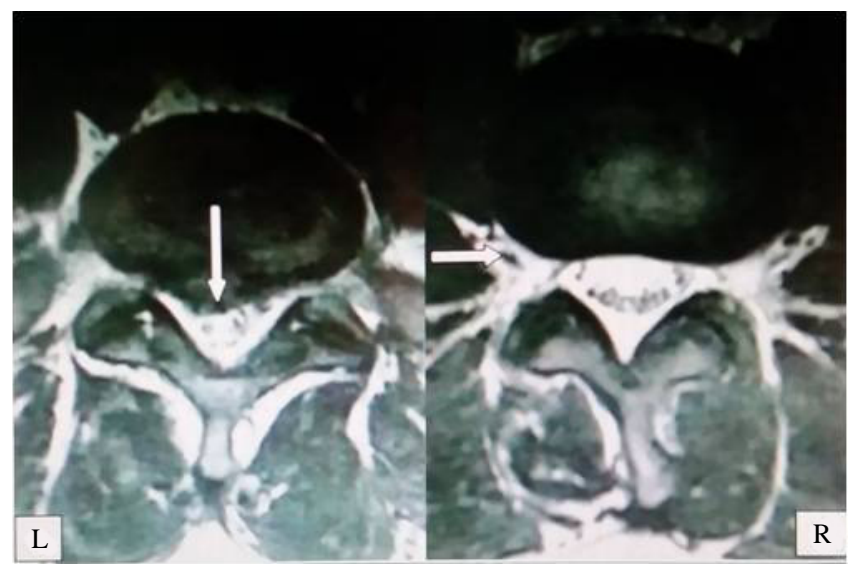

Figure 2. L: Lumbar disc herniation between $\mathrm{L}_{5}$ and $\mathrm{S}_{1}$ (arrow). The nerve root adjacent to the intervertebral foramen was clearly visible, and there was no formation of a nerve root sheath cyst; R: Transverse section of $\mathrm{L}_{4} / \mathrm{L}_{5}$. The water content of the lateral nerve root of the right intervertebral foramen was slightly increased (arrow).

[7] [8]. The operators know the inherent risks of performing injections and make every effort to avoid the risks including that drugs injected into the veins by accident, subarachnoid space injections, and injuries induced by organ punctures. The injurious consequences induced by straying into the cerebrospinal fluid are different due to different drug compositions [1] [2]. Local anesthetics may cause a higher level of nerve block, complete spinal anesthesia, and may even be life-threatening. Glucocorticoid suspensions are known to induce local or diffuse cerebral meningitis and arachnoiditis. In some serious cases, glucocorticoid suspensions may induce separation of the central ventricle or spinal cord aqueduct, which will lead to fatal intracranial hypertension [9]. Different agents of vitamin $B_{12}$ are used to inject into epidural space; however, it is unknown what would happen if vitamin $B_{12}$ was injected into the cerebrospinal fluid [10].

Inward-going intervertebral foramen puncture via the skin $5-7 \mathrm{~cm}$ outside away from the middle of thoracic or lumbar spine is the usual route to perform a paravertebral block. The target of drug injection is close to the intervertebral foramen, which can ensure satisfactory treatment. The inherent risks of the traditional route are injury to the nerve trunk, injection into the root sleeve, and puncture into the vertebral canal. In our hospital we use a different method to perform paravertebral injections. The puncture point is $1.5-2.0 \mathrm{~cm}$ side away to the middle of the lumbar spinous process. The needle is outward-going to reach the outside of the intervertebral foramen. Therefore, we assume that the injection target has a short distance to the intervertebral foramen. This method avoids drugs entering the subarachnoid space. The operators with different levels in our pain management center have treated more than 5000 cases using this method. The treatments were safe and effective, but there was nevertheless the complication reported herein.

The patient had bradycardia, hypotension, and high level paraplegia immediately after the injection in this case. High level paraplegia induced by the drug mixture was confirmed according to the immediate effects of treatment and recovery of neurologic function thereafter. A lumbar puncture was performed after $2 \mathrm{~h}$ and the cerebrospinal fluid was noted to be slightly turbid and light pink in color, which were consistent with the physical properties of the diluted mixture of triamcinolone acetonide acetate suspension and cyanocobalamin. Together, these findings indicate that a drug mixture was injected into the cerebrospinal fluid.

It is difficult to determine why the drug mixture was injected into the subarachnoid space. The radiologists, operators, and assistants described and analyzed the lumbar puncture procedure and the procedure was correct. We re-checked the injection point on the skin when the patient was sitting or lying on in the left lateral position. It is difficult to imagine the puncture needle went into the vertebral canal. The puncture needle may go outside of the vertebral canal. A MRI of the lumbar spine showed no clear root sleeve cyst in the patient. Moreover, the puncture needle went outward and was relatively far away from the intervertebral foramen. It is unlikely that the injection was performed from the $L_{5}$ nerve root sheath injection. We reviewed the procedure and considered that the needle may have entered into the cerebrospinal fluid via an upper nerve root cyst in the lever of the $L_{5} / S_{1}$ in- 
tervertebral foramen. Figure 2(L) shows the lumbar disc herniation at the level of $\mathrm{L}_{5} / \mathrm{S}_{1}$. Figure 2(R) shows that the water content of the lateral nerve root of the right intervertebral foramen was slightly increased at the level of $\mathrm{L}_{4}$ and $\mathrm{L}_{5}$. Although radiologists thought that the evidence was not sufficient to diagnose a nerve root cyst of $\mathrm{L}_{3}$ or $\mathrm{L}_{4}$, in combination with the lower pressure of the cerebrospinal fluid, we concluded that cyst collapse was induced by the leakage of cerebrospinal fluid in the cyst due to a local puncture injury. Because we did not see any cerebrospinal fluid when we withdrew the plunger of the syringe before injection and most of the drug mixture was located in the subarachnoid space, these data support the conclusion.

\section{Conclusion}

The current case provides two learning points. First, there is a risk that drugs may migrate into the cerebrospinal fluid even during outward-going paravertebral punctures. The possible mechanism may be a superior cyst in the nerve root or root sleeve. Second, the outcome after triamcinolone acetonide acetate suspension and cyanocobalamin incorrectly injected into the cerebrospinal fluid is not as serious as we had imagined. The patient had no any sequelae in the central nervous system after 6 months of follow-up. Indeed, longer follow-up is required in cases with any sequelae. However, we do not know if other patients will exhibit serious symptoms under the same conditions. We anticipate arriving at a definitive conclusion after conducting corollary studies using animal models.

\section{References}

[1] Mizuno, J., Gauss, T., Suzuki, M., et al. (2007) Encephalopathy and Rhabdomyolysis Induced by Iotrolan during Epiduroscopy. Canadian Journal of Anesthesia, 54, 49-53. http://dx.doi.org/10.1007/BF03021899

[2] Maugans, T.A. (2010) Intracranial Migration of a Fractured Intrathecal Catheter from a Baclofen Pump System: Case Report and Analysis of Possible Causes. Neurosurgery, 66, 319-322. http://dx.doi.org/10.1227/01.NEU.0000363183.78323.D7

[3] Manchikanti, L. and Hirsch, J. (2015) An Update on the Management of Chronic Lumbar Discogenic Pain. Pain Management, 5, 373-386. http://dx.doi.org/10.2217/pmt.15.33

[4] Simon, J., McAuliffe, M., Shamim, F., et al. (2014) Discogenic Low Back Pain. Physical Medicine and Rehabilitation Clinics of North America, 25, 305-317. http://dx.doi.org/10.1016/j.pmr.2014.01.006

[5] Torstensson, T., Lindgren, A. and Kristiansson, P. (2013) Improved Function in Women with Persistent PregnancyRelated Pelvic Pain after a Single Corticosteroid Injection to the Ischiadic Spine: A Randomized Double-Blind Controlled Trial. Physiotherapy: Theory and Practice, 29, 371-378. http://dx.doi.org/10.3109/09593985.2012.734009

[6] Cohen, S.P., Mao, J., Vu, T.N., et al. (2013) Does Pain Score in Response to a Standardized Subcutaneous Local Anesthetic Injection Predict Epidural Steroid Injection Outcomes in Patients with Lumbosacral Radiculopathy? A Prospective Correlational Study. Pain Medications, 14, 327-335. http://dx.doi.org/10.1111/pme.12027

[7] Çetin, M.F., Karaman, H., ÖlmezKavak, G., et al. (2012) Efficacy of Transforaminal Lumbar Epidural Steroid Injections in Patients with Lumbar Radiculopathy. A $\breve{g r l}$, 24, 77-84. http://dx.doi.org/10.5505/agri.2012.98704

[8] Manchikanti, L., Cash, K.A., McManus, C.D., et al. (2012) Fluoroscopic Caudal Epidural Injections with or without Steroids in Managing Pain of Lumbar Spinal Stenosis: One-Year Results of Randomized, Double-Blind, Active-Controlled Trial. Journal of Spinal Disorders \& Techniques, 25, 226-234. http://dx.doi.org/10.1097/BSD.0b013e3182160068

[9] Moon, J.H., Jung, H.W., Lee, H.C., et al. (2015) A Study of Experimental Autoimmune Encephalomyelitis in Dogs as a Disease Model for Canine Necrotizing Encephalitis. Journal of Veterinary Science, 16, 203-211. http://dx.doi.org/10.4142/jvs.2015.16.2.203

[10] Scalabrino, G., Veber, D. and Tredici, G. (2014) Relationships between Cobalamin, Epidermal Growth Factor, and Normal Prions in the Myelin Maintenance of Central Nervous System. International Journal of Biochemistry and Cell Biology, 55, 232-241. http://dx.doi.org/10.1016/j.biocel.2014.09.011 